\title{
Model-Driven Inquiry: Beyond Ethnography and Contextual Inquiry
}

\author{
Larry Constantine \\ Madeira Interactive Technologies Institute, \\ University of Madeira, Caminho da Penteada, \\ 9000-390 Funchal, Portugal \\ LConstantine@m-iti.org
}

Keywords: model-driven inquiry, user research, user requirements, contextual inquiry, ethnography.

\section{Extended Abstract}

Model-driven approaches are of growing influence in interaction design owing to the promise of yielding more orderly and manageable processes with enhanced traceability from initial conception and the establishment of requirements through to design and final realization. Model-driven inquiry is an agile technique, an accelerated alternative in its own right to contextual inquiry and other ethnographic approaches for user research, field study, and requirements gathering that can also be combined with these more conventional techniques.

Model-driven inquiry is a proven, industrial-strength method that can streamline and systematize user research. The approach enables projects to get the greatest benefit from the fewest resources by reducing the amount of time needed for adequate field investigation. The approach allows for focusing field inquiry more sharply and specifically by identifying specific areas for investigation based on risk, uncertainty, ambiguity, and impact on design outcomes. By drawing on the extant knowledge and insight of designers and identifying boundaries between the known and not-known, model-driven inquiry can dramatically reduce the need for extended observation or extensive user research. Although the approach evolved within interaction design practice, it also has potential application in academic research.

Participants in this tutorial will learn about the relationship of model-driven inquiry to contextual inquiry and other methods based in ethnography and participant observation. Through a mix of presentation, discussion, hands-on application to realworld problems, and review of experiences, participants will learn how to use models and modeling to drive inquiry, how to choose models for leverage in both inquiry and design, and how to formulate focused and efficient field research by selecting effective inquiry techniques. The relationship to and integration with contextual inquiry will also be addressed.

Model-driven inquiry, based on exploratory modeling and using techniques from joint essential modeling, has been used extensively in design practice and is taught in the joint Carnegie Mellon/University of Madeira MHCI program. 
Presentations and discussions will be combined with hands-on small-group exercises and a case study problem will enable participants to experience and trace the entire model-driven design process through to a preliminary design.

Topics to be covered in this tutorial include:

- nature and philosophy of ethnography and ethnographic methods

- overview of contextual inquiry

- model-driven inquiry, exploratory modeling, and joint essential modeling

- exploratory models: inventories, maps, and profiles

- activity and task modeling, basic definitions

- methods of generating inventory content:

- activities, roles, and tasks

- provisional drafts of activity profiles and role profiles

- user and professional participation in exploratory modeling

- compiling, clustering, and categorizing queries

- confidence sorting, salience sorting, and risk ranking of queries

- selecting appropriate inquiry methods and target informants

- incorporating inquiry findings to refine and elaborate models

- iterative inquiry for agile projects

- integrating model-driven inquiry with contextual inquiry

\section{References}

1. Constantine, L.L.: Model-Driven Inquiry. User Experience 8(3), 16-19 (2009)

2. Constantine, L.L.: Human Activity Modeling: Toward a Pragmatic Integration of Activity Theory and Usage-Centered Design. In: Seffah, A., Vanderdonckt, J., Desmarais, M. (eds.) Human-Centered Software Engineering II. Springer, NY (2008)

3. Constantine, L.L.: Beyond User-Centered Design and User Experience. Cutter IT Journal, 17(2) (February 2004)

4. Windl, H.: Usage-Centered Exploration: Speeding the Initial Process. In: Constantine, L. (ed.) Proceedings, for USE 2002: First International Conference on Usage-Centered, TaskCentered, and Performance-Centered Design, pp. 39-53. Ampersanda Press, Rowley (2002) 